\title{
Preference reversals between intertemporal choice and pricing
}

\author{
Peter D. Kvam (pkvam@ufl.edu) \\ Konstantina Sokratous (k.sokratous@uff.edu) \\ Gabriela Johnson (gab.johns199@ufl.edu) \\ Shu Ting Lin (shutinglin@ufl.edu) \\ Emily Unruh (emily.unruh@ufl.edu) \\ Department of Psychology, University of Florida \\ 945 Center Dr, Gainesville, FL 32611, USA
}

\begin{abstract}
Preference reversals in risky choice - where people favor lowrisk prospects in binary choice but assign higher prices to highrisk prospects - have led to models of response processes that differentiate pricing from choice. Theories of intertemporal choice do not distinguish between response processes, assuming instead that eliciting choices or prices will lead to the same inferences about people's preferences for delayed outcomes. Here, we show that this assumption is incorrect. Participants in a price-choice experiment showed systematic preferences for smaller-sooner (SS) over larger-later (LL) options in binary choice, but reversed this apparent preference by pricing the exact same LL options higher than the SS options. This reversal in pricing results in less impulsive behavior, suggesting that pricing frames may reduce choice impulsivity. To explain these diverging price and choice findings in a common framework, we propose a variant of a pricing model from risky choice that accommodates these effects.

Keywords: intertemporal choice; price; preference reversal; computational cognitive modeling
\end{abstract}

\section{Introduction}

Experimental and modeling work in risky decision making supports the claim that the method of eliciting preference whether a decision maker assigns a price to a pair of options separately or chooses between them directly - affects the apparent preference order between risky prospects (Lichtenstein \& Slovic, 1971, Slovic \& Lichtenstein, 1983). Participants are generally more inclined to choose the safest option when offered a choice between a "safe" lottery (e.g., 90\% chance of \$4) and a "risky" lottery (e.g., 30\% chance of \$16). Conversely, when participants are offered the chance to price these options by specifying how much they would be willing to pay to acquire it (WTP), how much they would be willing to accept to sell it (WTA), or what dollar value would be equivalent to each option (certainty equivalent), they tend to assign a higher price to the risky prospect than to the safe one. Selecting safe options (p-bet) to risky ones (\$-bet) when making a choice and then assigning a higher value to risky options over safe ones when pricing individually each option, is referred to as preference reversalsin risky choice.

Such conclusions are problematic for many of the current theories in risky choice, as they imply failure of procedure invariance, the idea that people should exhibit consistent preferences regardless the procedure used to measure their preference. Violations of procedure invariance led to the idea that preferences are constructed when an answer is prompted (Slovic, 1995), such that they are modified by the elicitation process or even do not exist until elicited (Busemeyer et al.
2020). However, a common representation of value underlying both choice and pricing is feasible, as shown by different theories that reconcile the idea of procedure invariance in terms of a common utility representation (Johnson \& Busemeyer, 2005). True subjective values remain therefore unchanged, but the way people respond to different elicitations change between decision and pricing contexts. Most effective explanations have been based on the ideas of anchoring and adjustment (Tversky et al., 1990, Cubitt et al., 2004), or on describing choice and pricing as dynamic, stochastic processes (Johnson \& Busemeyer, 2005, Kvam \& Busemeyer. 2020).

Despite extensive documentation of preference reversals in risky choice, relatively little attention has been paid to the potential of reversals in other domains, such as intertemporal choice. Indeed, "preference reversals" refer to a different phenomenon in delay discounting, where people shift from choosing one delayed prospect $\left(x_{1}, t_{1}\right)$ over another $\left(x_{2}, t_{2}\right)$ when a fixed delay is added to both of them $\left(x_{1}, t_{1}+\Delta t\right.$ vs $x_{2}$, $\left.t_{2}+\Delta t\right)$. While these types of preference reversals are important to understanding the functional form of delay discounting (Mazur. 1987), they may have obfuscated the possibility or prevalence of choice-price preference reversals in intertemporal choice where research mainly focus on reversals that are induced by modifications of the stimuli, as opposed to modifying the method of eliciting preference (Loewenstein \& Thaler, 1989).

Although choice-price reversals have not seen considerable scrutiny in intertemporal choice, violations of procedure invariance still appear. Hardisty et al. (2011) reviewed three types of intertemporal choice paradigms where discounting rates may be elicited by either choice-based approaches (indifference points inferred from binary choice), matching (discount rates inferred from the SS value equated to the LL value), and adaptive staircase procedures that titrate the value or delay of the options until choices made in sequence switch between favoring the LL and SS option. Each of these methods can reach different conclusions about the discounting rates that participants are using to assess the value of delayed prospects, suggesting that the apparent values that participants assign to their options are sensitive to how preferences is measured (see also Frederick et al., 2002, for a review). In principle, this suggests that intertemporal choice may be suceptible to the same sorts of (apparent) violations of procedure invariance that we find in risky choice. 
Previous work in behavior analysis has shown parallels between risky and intertemporal choice, in some cases modeling probability discounting and delay discounting as overlapping (if not entirely common) processes (Green \& Myerson, 2004). Although the cognitive mechanisms involved in risky choice and intertemporal choice appear dissociable, they coincide much of the time (Myerson et al. 2011). If the same mechanisms that drive preference reversals in risky choice / probability discounting are also present in intertemporal choice / delay discounting, it stands to reason that there should be choice-price reversals in intertemporal choice. Preliminary support for this hypothesis comes from work seeking to understand violations of procedure invariance by Tversky et al. (1990, Table 4), which hinted at the possibility of preference reversals between price and choice in delayed outcomes. Tversky et al. showed reversals between a few example prospects: participants would choose the option to receive $\$ 2500$ in 5 years rather than $\$ 3550$ in 10 years when given a choice between the two, but would set a higher willingness to accept (WTA; the lowest immediate cash payment at which they would be willing to give up the delayed prospect) on $\$ 3550$ in 10 years than on $\$ 2500$ in 5 years. Due to the large sums of money involved, these choices were not actually carried out, but Tversky et al. were still able to show that over 50 percent of their participants appeared to switch from preferring the smaller, sooner (SS) option in choice to the larger, later (LL) option in pricing.

The implications for research on delay discounting are substantial. Early work on intertemporal choice, including some of the seminal work on models like the hyperbolic discounting model, commonly elicited dollar values such as price equivalents to identify indifference points for delayed outcomes (Kirby \& Maraković, 1995), whereas later work has focused almost exclusively on binary choices between prospects (Bickel \& Marsch, 2001). As we suggest below, the apparent reversals between these two types of response processes suggest that early participants may have been perceived as less impulsive than later ones.

An important distinction to be made between risky and intertemporal choice regards risk-aversion, which is typically seen as a socially desirable outcome, at least in the sense that "disordered" decision making and problem gambling involves frequently taking low-probability or unfavorable gambles (Ciccarelli et al., 2020). In a risky decision making scenario, asking an individual to engage in pricing will more likely exacerbate these issues, causing a participant to favor risky prospects (\$-bets) even more strongly. A price-focused intervention would not be suitable for reducing risk-taking behavior or disordered gambling, but a similar manipulation may be successful in intertemporal choice. A multitude of behaviors connected to negative health outcomes (such as substance use) are related to choice impulsivity, defined as a propensity to favor the smaller, sooner outcome in intertemporal choice (Rung \& Madden, 2018). Despite this, interventions to reduce discounting have proven troublesome to develop due to small effect sizes or practical challenges to implementation (Rung et al. 2019). If we can show that eliciting intertemporal preferences through pricing as opposed to choice consistently shifts responses toward the larger-later option - as we should suspect given previous findings (Tversky et al., 1990) - then it may be possible to develop simple and effective interventions that leverage choice-price reversals to reduce impulsive choice.

In the following section, we will describe an experiment which aimed to explore the robustness of choice-price preference reversals across a wide variety of intertemporal prospects. Understanding reversals and their prevalence can determine both how important they are to accommodate within theories of value, as well as how effective interventions focusing on pricing might be.

\section{Methods}

To understand the prevalence and causes of choice-price reversals in intertemporal choice, we ran an experiment that manipulated the response format -- binary choice and willingness-to-pay judgments - within subjects. A total of 46 participants were recruited through Prolific, receiving the amount of $\$ 10$ per hour for participation and an additional bonus of up to $\$ 5$, based on the responses they provided.

Our experiment diverged from previous studies (Tversky et al., 1990, which used huge payoffs and delays that were purely hypothetical) in that participants received some of the delayed payoffs, and the payoffs were sums of money most participants could reasonably expect to encounter on a regular basis. To emphasize the importance of preference orders, participants were rewarded based on their choices and their order of pricing for individual delayed prospects, such that they were more likely to receive delayed prospects to which they assigned a higher price. Participants were awarded with a delayed bonus (1-3 days later) based on which of four individual delayed prospects the assigned the highest price (participants were not told which delayed prospects these would be) or received one of the delayed prospects that they chose in one of the decision trials. The payoffs that participants could receive in this payment scheme consisted of \$1-5 and delays of 1-3 days, although the range of payoffs and delays across the experiment were larger.

The experiment took approximately 60 minutes to complete. Of the 46 participants, we removed 8 for failing attention checks, selecting dominated response options in the choice trials (e.g., selecting $\$ 8$ in 10 weeks over $\$ 12$ today), or assigning prices to delayed prospects that were more than $50 \%$ higher than the actual outcome on a total of at least two occasions. Out of the 38 remaining participants, there were 20 men and 18 women who completed the experiment successfully. The average age of our participants was 32.82 years $(S D=12.72)$.

During the experiment, subjects were presented with a total of 88 choice trials and 175 pricing trials, with choice and price trials in separate blocks. The 88 choice pairs consisted 


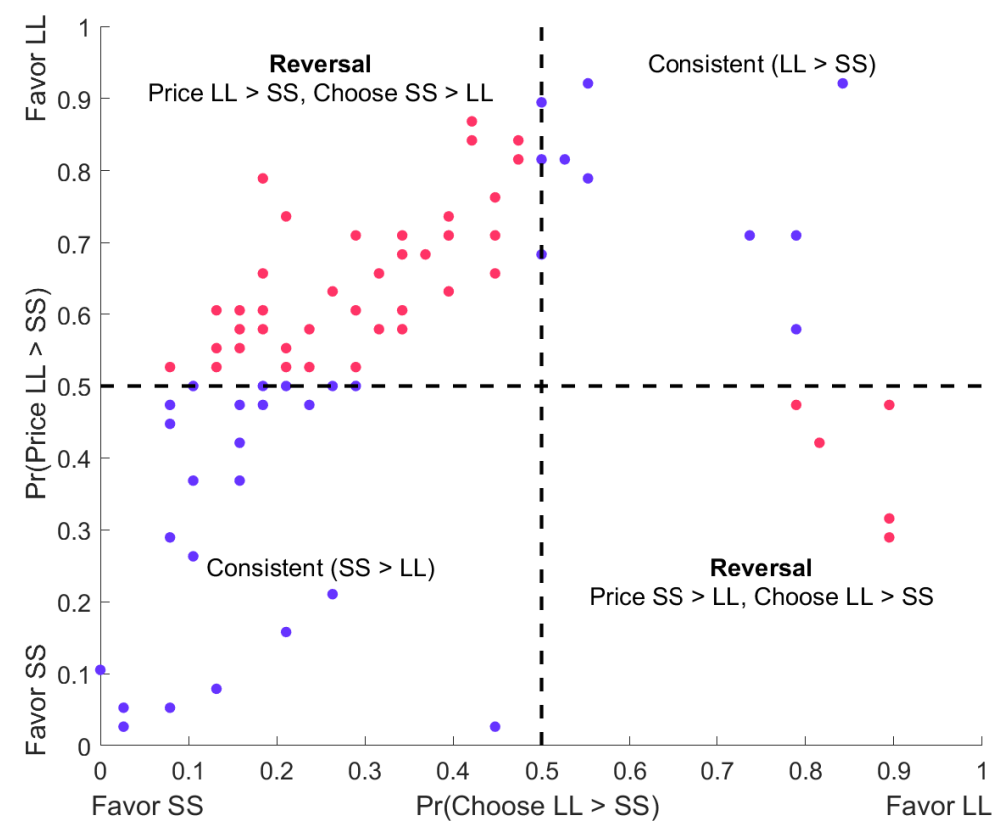

Figure 1: Scatter plot of the proportion of times participants chose the larger, later [LL] option over the smaller, sooner [SS] option (x) against the proportion of times they priced the LL option higher than the SS option (y).

of 176 unique options paired together, including one SS option that had an immediate payoff (\$1.50, Today). Aside from the lone option with an immediate payoff, each of these unique options was also shown in the pricing trials. The payoffs for all of the delayed prospects varied from \$0-30, and the delays varied from 0-365 days. In Qualtrics, the choice trials consisted of two stimuli each, presented side by side, while the pricing trials were presented as a single stimulus with a sliding scale to enter price. With the exception of a few catch trials that used dominated prospects, each choice trial included one shorter-sooner (SS) and one larger-later stimulus (LL). The stimuli were randomly shuffled on the left and right sides of the screen, to avoid participants developing a bias toward one side or the other. The order of the stimuli was also randomized within each block of trials.

For example, a choice trial might include an SS option of receiving \$15 in 40 days or an LL option of receiving \$20 in 70 days. Subjects would indicate which option they preferred by simply pressing the left or right mouse button to select the respective prospect. For the pricing trials, participants entered their willingness to pay (buying) prices using a slider tool on Qualtrics that ranged from \$0 (the minimum payoff in the experiment) to $\$ 30$ (the maximum payoff across all trials of the experiment). In the pricing trials, only one stimulus was presented at a time.

Since all the stimuli individually presented during pricing trials were paired and included in the choice trials, we were able to obtain a separate/individual price for every prospect presented as a choice option, which allowed us to observe whether participants consistently priced and chose one type of stimuli over the other (e.g SS over LL) or if instead they engaged in choice-price preference reversal.

\section{Results}

A total of 46 participants completed the experiment, but only 38 were retained due to the 8 others failing multiple attention checks. We used a Bayesian approach to estimate the proportions of participants that preferred the LL to the SS option on each choice pair, and the proportion of participants who priced the LL option higher than the corresponding SS option in the price trials. The first analysis was programmed in JAGS, and looked at the overall rates of selecting the LL over SS option by estimating the proportion parameter of a Bernoulli distribution. For this proportion parameter, a simple uninformative uniform distribution $U(0,1)$ was used for the prior. Here, we report the posterior mean estimate of this proportion alongside the $95 \%$ Highest Density Interval [HDI], which indicates the $95 \%$ most likely values of the overall tendency to select the LL over the SS option.

Across all participants, choice pairs, and trials, the decisions that participants made in the choice condition consistently favored the SS option, $M\left(\operatorname{Pr}_{\text {choice }}(L L>S S)\right)=$ $.32,95 \%$ HDI $=[.23, .42]$ indicating a general tendency toward choice impulsivity in our sample. Conversely, the order of price responses largely favored the LL option, $M\left(\operatorname{Pr}_{\text {price }}(L L>S S)=.54,95 \% \mathrm{HDI}=[.44, .65]\right.$. The rate of favoring the LL option in price trials was quite substantially and credibly higher than the choice condition, with the contrast between price and choice $M\left(\operatorname{Pr}_{\text {price }}(L L>S S)-\right.$ 


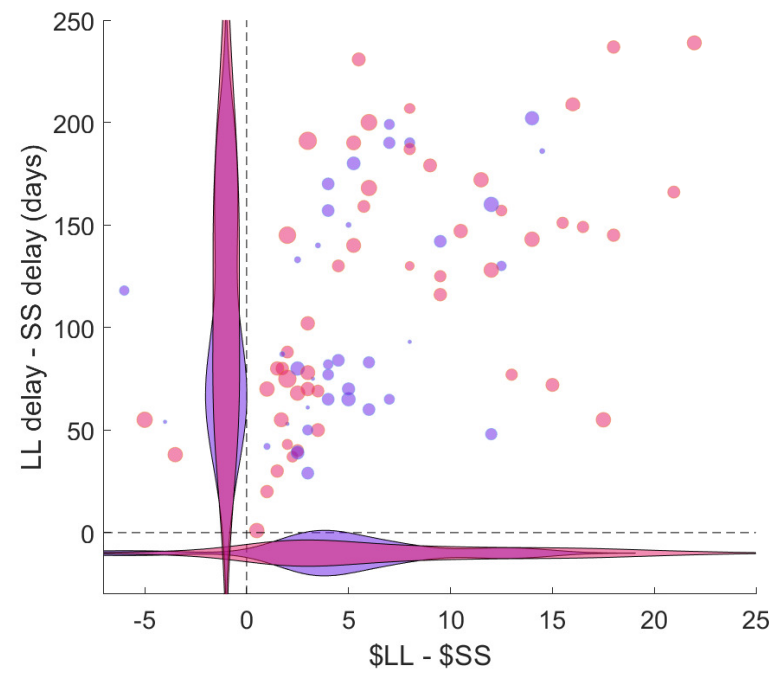

Figure 2: Scatter plot of the instances of preference reversals (red dots) and consistent preferences (blue dots) as a function of the difference in price between the LL and SS option (xaxis) and the difference in delay between LL and SS options (y-axis). The size of each dot corresponds to the magnitude of the difference between pricing and choice conditions, and the density plots (shaded red / blue regions along each axis) correspond to the relative frequency of reversals or consistent choices (respectively) across the range of stimuli.

$\left.\operatorname{Pr}_{\text {choice }}(L L>S S)\right)=.22,95 \%$ HDI $=[.08, .36]$.

From these results, it is clear that there are consistent choice-price preference reversals, favoring the SS option in choice but the LL option in pricing. However, there was also substantial variation in behavior across the different choice pairs. This can be seen in Figure 1, which shows the proportion of participants favoring the LL option in the choice trials on the $\mathrm{x}$ axis and the proportion of participants favoring the LL option in the price trials on the y axis. Despite this, with a few exceptions, there was generally a positive relationship between the proportion of participants favoring the LL option in choice and the proportion favoring the LL option in pricing (Pearson $r=0.38$, Spearman $\rho=0.54$ ). As we suggest below in the model outline, this indicates that there may still be a consistent value representation that is common to both choice and price, but that the methods of eliciting responses in the two conditions lead to diverging preference orders.

As a result of reversals of preference, our inferences about discounting rates in each of our participants will differ based on whether we use choices to estimate them or prices. We fit a hyperbolic discounting model to the choice data and to the pricing data using a hierarchical Bayesian implementation of the hyperbolic discounting model (Molloy et al. 2020, Kvam et al., n.d.). For the choice data, the group-level estimate of the discounting rate was $\log (k)=-0.04$ (95\% HDI $=$ $[-0.15,0.10])$. For the price data, the group-level estimate of the discounting rate was significantly lower, $\log (k)=-7.14$
$(95 \% \mathrm{HDI}=[-7.96,-2.83])$. As a result, the conclusions we draw from the choice data indicate much steeper discounting / greater impulsivity than those we would draw from analyzing the pricing data using traditional delay discounting models.

To examine what characteristics of the choice pairs led to reversals, we examined behavior on each pair of options individually. An analysis of how the differences in payoffs and delays were related to the rates of preference reversals is shown in Figure 2 Generally, very large differences in payoffs or delays tended to lead to preference reversals between choice and price conditions (greater density of red dots at high payoff differences and high delay differences), while choices and prices were most consistent when the payoff differences was around \$2-8 and delay differences were around 20-100 days. The further outside these ranges that the stimuli crept, the more common choice-price preference reversals were.

Four illustrative examples are shown in Figure 3 Choices and prices tended to be most consistent when there was a relatively small difference in the attributes of the options (left panel) or when there was a clear "better" option (middle left panel). The most common type of reversal, where the SS option was chosen but the LL option was priced higher, tended to manifest when there was a substantial gap between the payoffs of the SS and LL option but where the direct comparison was still somewhat difficult or slightly favorable to the SS option (middle right panel). These reversals heavily drove the overall effect reported above, as they constituted the largest overall portion of results (pink dots in the top left of Figure 1 out-number those in any other quadrant).

Although much more rare, reversals also occurred in the other direction, where participants priced the SS option higher, but chose the LL option when placed side by side. Since this pair of outcomes were only observed on 5 out of the 88 pairs, it is difficult to deduce systematically why they occur as the amount information is constrained, but it seems that they take place most often when both the SS and LL option have high outcomes and relatively short delays (right of Figure 3).

\section{Model}

As with choice-price reversals in risky decision making, the most common models of intertemporal choice are unable to handle this pattern of results. This is because the same model is typically used to account for both pricing and choice prospect theory being the most popular in risky choice (Kahneman \& Tversky, 1979) and hyperbolic discounting in intertemporal choice (Mazur, 1987). In hyperbolic discounting, the subjective value of a delayed prospect is calculated by "discounting" its objective payoff according to a subjective weight determined by the amount of time a person must wait before they can receive it. Formally, the value of a delayed prospect, $v(x, t)$ where $x$ is the amount a person could receive and $t$ is the time at which they would receive it, is calculated as $v(x, t)=\frac{x}{1+k t}$. The discounting rate $k$ determines how 


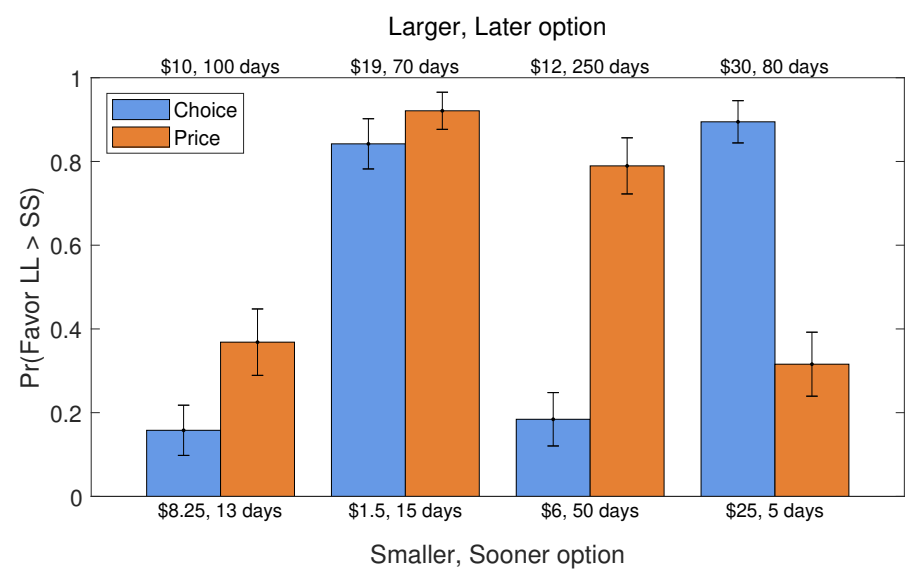

Figure 3: Examples of pairs of intertemporal prospects used in the experiments and the resulting preferences in the choice (blue) and price (orange) conditions. The pair on the left (smaller sooner [SS] bottom, larger later [LL] top) yielded consistent preferences for the SS option, while the pair second from the left yielded consistent preferences for the LL option. Conversely, the pair second from the right yielded the most common type of preference reversal where the SS option was chosen in the binary choice condition but the LL option was assigned a higher price more often, and the pair on the right yielded a less common type of reversal where the SS option was assigned a higher price more often but the LL option was selected when the two prospects were shown together.

much a payoff decreases in subjective value as it is pushed further away in time. A participant should typically assign $v(x, t)$ (with some error) as the price of a delayed prospect, and on average will choose a delayed prospect $\left(x_{1}, t_{1}\right)$ over another delayed prospect $\left(x_{2}, t_{2}\right)$ as long as $v\left(x_{1}, t_{1}\right)>v\left(x_{2}, t_{2}\right)$. Typically, a choice proportion is derived by including a logistic or softmax choice rule, such that the probability of choosing an LL over an SS option is

$$
\operatorname{Pr}(L L \succ S S)=\frac{1}{1+\exp (m[\cdot v(L L)-v(S S)]} .
$$

The failure to predict a reversals comes from the fact that a person will tend to both price the SS (LL) higher and choose the SS (LL) anytime $v(S S)>v(L L)$. On a more fundamental level, the problem with the hyperbolic discounting model (and nearly all other existing intertemporal choice models) is that they posit a static, deterministic account of value that is supposed to be common to any method of eliciting preference. To account for reversals, we instead have to look to dynamic and stochastic models that provide an account of how elicitation (choice, price) affects apparent preference orders (Johnson \& Busemeyer, 2005).

To demonstrate how to account for reversals using more nuanced models, we implement an adapted version of the price accumulation model developed by Kvam \& Busemeyer (2020). For simplicity, let us assume that choice proportions are computed exactly as in Equation 1 and focus on developing a model of price. Although the robust preference reversals necessitate a shift in how we model intertemporal pricing, a new model that accommodates these findings is fortunately not too difficult to construct. The only modification we make to the price accumulation model is to assume that the accumulation process is driven by the payoff and delay rather than the payoff and probability. Formally, we assume that the probability of stepping toward the payoff is $p=\frac{1}{1+k t}$ and the probability of stepping toward $\$ 0$ (the immediate payoff) is $1-p=\frac{k t}{1+k t}$.

A diagram of this model and its predictions is shown in Figure 4. In this approach, a price is generated as a participant thinks about the attributes of the delayed prospect and assesses its value. A participant making a price response first anchors on the outcome of the delayed prospect (as in anchoring \& adjustment Goldstein \& Einhorn, 1987), generating a "start point" for the price accumulation process. Formally, the location of a person's start point is specified by a proportion $q$ specifying how much a person uses the anchor (the polar angle of their start point is $\phi=\frac{\pi \cdot \$_{\max }}{2} \cdot q \cdot x$, where $x$ is the payoff of the delayed prospect and $\$_{\max }$ is the upper anchor of the price scale. The strength of this anchor is given by another free parameter $s_{v}$ specifying the distance (polar radius) of the starting point from zero. The $x$ and $y$ Cartesian coordinates of the starting point $s$ are given as $s=\left[s_{\nu} \cos (\phi), s_{\nu} \sin (\phi)\right]$. This creates an initial state that is biased toward high prices for an LL option and biased toward low prices for an LL option, and which changes based on the perspective of the participant (e.g., higher for sellers and lower for buyers).

From this starting point, the participant then considers the payoff of the delayed prospect and the time they would have to wait to receive it. Thinking the payoff drives them toward higher prices, resulting in their state $s$ moving in the direction of the payout $x$, while thinking of the delay drives them toward lower prices, resulting in their state $s$ moving in the direction of $\$ 0$ (what they will receive before the delay elapses). We formalize this accumulation process $y$ assuming that this 


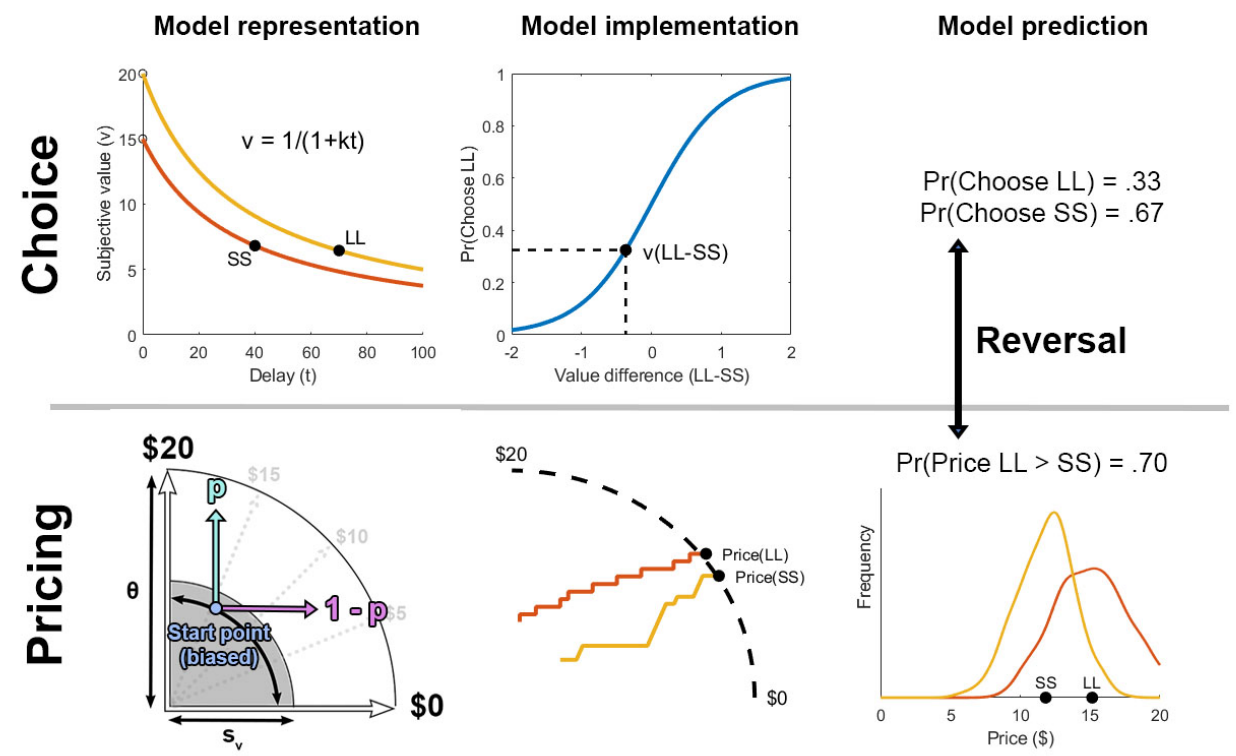

Figure 4: Example of how the response processes involved in choice (top) and pricing (bottom) can lead to diverging apparent preferences between SS and LL options.

accumulation process is driven by the same valuation process as choice - in this case a hyperbolic function. Formally, the probability $p$ of moving toward the payoff at each step is $\frac{1}{1+k t}$ where $t$ is the delay and $k$ is the discounting rate. Conversely, the probability of stepping toward $\$ 0$ at each step is $1-p=1-\frac{1}{1+k t}=\frac{k t}{1+k t}$. The accumulation process unfolds as the participant steps in either direction at each moment in time, forming an accumulation trajectory like the one shown in the bottom middle panel of Figure 4

A participant continues this accumulation process until they obtain sufficient support for a price on the scale, where one price $j$ on the continuum meets the condition $\operatorname{comp}_{j}(s) \geq$ $\theta$. The parameter $\theta$ controls how much support a price requires before it is selected, and creates a quarter-circular boundary to the price accumulation process as shown in Figure 4 A higher value of $\theta$ will result in a longer accumulation process but one that yields prices closer to the hyperbolic discounting function, while a lower value of $\theta$ will result in a shorter accumulation process that yields prices that are strongly influenced by the initial point / anchor.

The complete price accumulation process specified by this model will produce a joint distribution of prices and response times (Kvam \& Busemeyer, 2020), but for our present purposes we only simulate the price distributions. Figure 4 shows an example where a person is pricing or deciding between an SS option (\$15, 40 days) and an LL option $(\$ 20,70$ days). Setting the free parameters of the model to $k=.03$, $\theta=1.5, s_{v}=.5$, and $q=1$ in the price model and $k=.03$ and $m=2$ in the choice model (top panels) results in a distribution of prices that favors the LL option (bottom right of Figure 4 ) but choice proportions favoring the SS option (top right of
Figure 47. This illustrates how the same value representation ( $k=.03$ in both models) can lead to diverging preferences in choice and pricing due to the SS / LL anchors in pricing (consistent with the explanation posed by Tversky et al., 1990) and hinting that our pattern of results can be captured by this modified price accumulation model.

\section{Discussion}

When first discovered, preference reversals between pricing and choice in risky decision making were viewed as major violations of procedural invariance, where apparently different representations of value seemed to be used when people chose between options or assigned prices to them individually. Since then, several models of the pricing process have been proposed that can account for reversals in observed preference orders while still using the same underlying valuation process as decision making models (Johnson \& Busemeyer. 2005). Despite widening use, the concepts proposed in these models have not yet made it into the realm of intertemporal choice modeling.

Although this approach to modeling provides an explanation for why preference reversals occur in principle, a complete account of choice-price reversals in intertemporal valuation ought to model individual-level decisions and prices. The next step in developing these models of intertemporal choice and pricing will estimate individual-level parameters, and connect the two elicitation methods to a common set of latent processes (as in Kvam \& Busemeyer. 2020).

Naturally, our approach does not provide a complete account of all mechanisms that could have created preference reversals between choice and price. One factor that may be 
at play is that pricing trials show each option separately, potentially influencing the "anchors" that participants generate for their responses (Rottenstreich \& Tversky, 1997; Frederick et al., 2002). This influence of context could be disentangled from the elicitation method in future work by displaying a pair of options on the screen (as in the choice condition) but having participants only price one of them.

Here, we took the first steps toward a deeper understanding of choice-price reversals in intertemporal choice, showing that they are both widespread and systematic. In some ways this phenomenon is positive: although taking a pricing approach to measuring risk preferences leads to greater risktaking, taking a pricing approach to measuring delay preferences results in "shallower" discounting. Shifting choices toward larger-later options over smaller-sooner ones is a potential route to reductions in choice impulsivity more generally. This can be done simply by phrasing a delay discounting problem as one of value (price them individually, then compare prices) instead as one of choice (selecting between them directly). It is possible that this could be leveraged in interventions attempting to manipulate and reduce delay discounting as a mechanism related to problems like substance use (Rung \& Madden, 2018).

\section{References}

Bickel, W. K., \& Marsch, L. A. (2001). Toward a behavioral economic understanding of drug dependence: Delay discounting processes. Addiction, 96(1), 73-86.

Busemeyer, J. R., Kvam, P. D., \& Pleskac, T. J. (2020). Comparison of markov versus quantum dynamical models of human decision making. Wiley Interdisciplinary Reviews: Cognitive Science, 11(4), e1526.

Ciccarelli, M., Griffiths, M. D., Cosenza, M., Nigro, G., \& D’Olimpio, F. (2020). Disordered gambling and attentional bias: The mediating role of risk-taking. Journal of affective disorders, 272, 496-500.

Cubitt, R. P., Munro, A., \& Starmer, C. (2004). Testing explanations of preference reversal. The Economic Journal, 114(497), 709-726.

Frederick, S., Loewenstein, G., \& O'donoghue, T. (2002). Time discounting and time preference: A critical review. Journal of economic literature, 40(2), 351-401.

Goldstein, W. M., \& Einhorn, H. J. (1987). Expression theory and the preference reversal phenomena. Psychological Review, 94(2), 236.

Green, L., \& Myerson, J. (2004). A discounting framework for choice with delayed and probabilistic rewards. Psychological Bulletin, 130(5), 769.

Hardisty, D. J., Fox-Glassman, K., Krantz, D., \& Weber, E. U. (2011). How to measure discount rates? an experimental comparison of three methods. An Experimental Comparison of Three Methods (November 1, 2011).

Johnson, J. G., \& Busemeyer, J. R. (2005). A dynamic, stochastic, computational model of preference reversal phenomena. Psychological Review, 112(4), 841-861.
Kahneman, D., \& Tversky, A. (1979). Prospect theory: An analysis of decision under risk. Econometrica, 47(2), 263292.

Kirby, K. N., \& Maraković, N. N. (1995). Modeling myopic decisions: Evidence for hyperbolic delay-discounting within subjects and amounts. Organizational Behavior and Human decision processes, 64(1), 22-30.

Kvam, P. D., \& Busemeyer, J. R. (2020). A distributional and dynamic theory of pricing and preference. Psychological Review, 127, 1053-1078.

Kvam, P. D., Romeu, R. J., Turner, B. M., Vassileva, J., \& Busemeyer, J. R. (n.d.). Testing the factor structure underlying behavior using joint cognitive models: Impulsivity in delay discounting and cambridge gambling tasks. Psychological methods, 26(1), 18-37.

Lichtenstein, S., \& Slovic, P. (1971). Reversals of preference between bids and choices in gambling decisions. Journal of Experimental Psychology, 89(1), 46-55.

Loewenstein, G., \& Thaler, R. H. (1989). Anomalies: intertemporal choice. Journal of Economic perspectives, 3(4), 181-193.

Mazur, J. E. (1987). An adjusting procedure for studying delayed reinforcement. Commons, ML.; Mazur, JE.; Nevin, $J A, 55-73$.

Molloy, M. F., Romeu, R. J., Kvam, P. D., Finn, P. R., Busemeyer, J., \& Turner, B. M. (2020). Hierarchies improve individual assessment of temporal discounting behavior. Decision, 7, 212-224.

Myerson, J., Green, L., \& Morris, J. (2011). Modeling the effect of reward amount on probability discounting. Journal of the Experimental analysis of behavior, 95(2), 175-187.

Rottenstreich, Y., \& Tversky, a. (1997). Unpacking, repacking, and anchoring: Advances in support theory. Psychological Review, 104(2), 406-15. doi: 10.1037/0033-295X .104 .2 .406

Rung, J. M., \& Madden, G. J. (2018). Experimental reductions of delay discounting and impulsive choice: A systematic review and meta-analysis. Journal of Experimental Psychology: General, 147(9), 1349.

Rung, J. M., Peck, S., Hinnenkamp, J. E., Preston, E., \& Madden, G. J. (2019). Changing delay discounting and impulsive choice: Implications for addictions, prevention, and human health. Perspectives on Behavior Science, 42(3), 397-417.

Slovic, P. (1995). The construction of preference. American psychologist, 50(5), 364-371.

Slovic, P., \& Lichtenstein, S. (1983). Preference reversals: A broader perspective. The American Economic Review, 73(4), 596-605.

Tversky, A., Slovic, P., \& Kahneman, D. (1990). The causes of preference reversal. The American Economic Review, 204-217. 